\title{
GPR17 mediates ischemia-like neuronal injury via microglial activation
}

\author{
BING ZHAO $^{1 *}$, HAO WANG $^{2 *}$, CAI-XIA LI ${ }^{1}$, SHENG-WEN SONG ${ }^{1}$, \\ SAN-HUA FANG ${ }^{3}$, ER-QING WEI ${ }^{3}$ and QIAO-JUAN SHI ${ }^{4}$
}

\begin{abstract}
${ }^{1}$ Department of Anesthesiology, The First Affiliated Hospital, School of Medicine, Zhejiang University, Hangzhou, Zhejiang 310003; ${ }^{2}$ Department of Neurology, Tongde Hospital of Zhejiang Province, Hangzhou, Zhejiang 310012; ${ }^{3}$ Department of Pharmacology, School of Medicine, Zhejiang University, Hangzhou, Zhejiang 310058;

${ }^{4}$ Experimental Animal Center, Zhejiang Academy of Medical Sciences, Hangzhou, Zhejiang 310013, P.R. China
\end{abstract}

Received February 24, 2018; Accepted August 23, 2018

DOI: $10.3892 / \mathrm{ijmm} .2018 .3848$

\begin{abstract}
GPR17 is a G (i)-coupled dual receptor, linked to $\mathrm{P} 2 \mathrm{Y}$ and CysLT receptors stimulated by uracil nucleotides and cysteinyl leukotrienes, respectively. Recent evidence has demonstrated that GPR17 inhibition ameliorates the progression of cerebral ischemic injury by regulating neuronal death and microglial activation. The present study aimed to assess the detailed regulatory roles of this receptor in oxygen-glucose deprivation/recovery (OGD/R)-induced ischemia-like injury in vitro and explore the underlying mechanism. The results demonstrated that $\mathrm{OGD} / \mathrm{R}$ induced ischemic neuronal injury and microglial activation, including enhanced phagocytosis and increased inflammatory cytokine release in neuron-glial mixed cultures of cortical cells. GPR17 upregulation during OGD/R was spatially and temporally correlated with neuronal injury and microglial activation. In addition, GPR17 knockdown inhibited OGD/R-induced responses in neuron-glial mixed cultures. GPR17 knockdown also attenuated cell injury induced by the agonist leukotriene $\mathrm{D}_{4}\left(\mathrm{LTD}_{4}\right)$ or uridine 5'-diphosphate (UDP) in neuron-glial mixed cultures. However, GPR17 knockdown did not affect OGD/R-induced ischemic neuronal injury in primary cultures of neurons. In primary astrocyte cultures, neither GPR17 nor OGD/R induced injury. By contrast, GPR17 knockdown ameliorated OGD/R-induced microglial activation, boosting phagocytosis and inflammatory cytokine release in primary microglia cultures. Finally, the results demonstrated that the conditioned medium of microglia pretreated with $\mathrm{OGD} / \mathrm{R}$ induced
\end{abstract}

Correspondence to: Dr Qiao-Juan Shi, Experimental Animal Center, Zhejiang Academy of Medical Sciences, 182 Tian-Mu-Shan Road, Hangzhou, Zhejiang 310013, P.R. China

E-mail: shiqiaojuan@163.com

*Contributed equally

Key words: $\mathrm{G}$ protein-coupled receptor 17, oxygen-glucose deprivation/recovery, neuron, microglia, small interfering RNA neuronal death, and the neuronal injury was significantly inhibited by GPR17 knockdown. These findings suggested that GPR17 may mediate ischemia-like neuronal injury and microglial activation in vitro; however, the protective effects on ischemic neuronal injury might depend upon microglial activation. Whether GPR17 regulates neuronal injury mediated by oligodendrocyte linkage remains to be investigated.

\section{Introduction}

Nucleotides, including extracellular adenine (ATP and ADP) and uracil (UTP, UDP, UDP-glucose and UDP-galactose) nucleotides, are signaling molecules involved in a wide range of physiological processes in response to pathological stimuli and cellular injury $(1,2)$. These actions are mediated by seven ionotropic $(\mathrm{P} 2 \mathrm{X})$ and eight metabotropic $(\mathrm{P} 2 \mathrm{Y})$ receptor subtypes. On the other hand, cysteinyl leukotrienes (CysLTs), including $\mathrm{LTC}_{4}, \mathrm{LTD}_{4}$ and $\mathrm{LTE}_{4}$, are potent proinflammatory mediators. CysLTs are implicated in diverse pathologies, such as respiratory diseases, and inflammatory conditions, including cardiovascular, gastrointestinal, and immune disorders, as well as neurodegenerative responses, which are mediated by CysLT receptor 1 and CysLT receptor 2 (3-7). Both nucleotides and CysLTs are proven inducers of brain inflammation, and have important roles in ischemic/inflammatory conditions and neurodegenerative responses $(2,7)$.

Currently, G protein-coupled receptor 17 (GPR17) has been described as a G (i)-coupled dual receptor linked to P2Y and CysLT receptors for uracil nucleotides and CysLTs, respectively (8-11). GPR17 is highly expressed in brain, heart, and kidney tissues undergoing ischemic injury $(9,12)$. In the brain, GPR17 is expressed in neurons, oligodendrocyte precursor cells (OPCs), and ependymal cells, but not in astrocytes, under physiological conditions (9,13-16). Following ischemic, traumatic, or demyelinating diseases, GPR17 is transiently and sequentially upregulated in neurons, microglia/macrophages, and oligodendrocyte precursors (9,14,17-19). Mounting evidence highlights the role of GPR17 in modulating oligodendrocyte precursor maturation, and GPR 17 is considered a novel target for innovative therapeutic approaches to demyelinating diseases, such as multiple sclerosis (19-23). 
Increasing evidence confirms that GPR17 is also involved in a broad range of pathological processes, such as cerebral ischemic injury and spinal cord damage $(9,14,17,18)$. In acute ischemic stroke, GPR17 upregulation in neurons is associated with enhanced cell death, while its knockdown markedly attenuates ischemic damage $(9,14,17)$. Beside its role in neuronal injury, GPR17 is a key player in brain remodeling and repair. In chronic ischemic stroke, activated microglia/macrophages induce GPR17 upregulation, and its inhibition prevents the progression of chronic ischemic injury by reducing brain atrophy while ameliorating chronic neuronal death and microgliosis $(14,17)$. Similar expression and modulation of GPR17 were demonstrated in traumatic spinal cord lesions (18). Thus, these findings indicated that GPR 17 might become a new therapeutic target in neurodegenerative diseases.

However, whether ischemia-like neuronal injury and microglial activation in vitro are mediated by GPR17 activation remains unclear. A better understanding of the related mechanism is crucial for developing effective therapeutics for ischemic stroke. The present study comprehensively determined the role of GPR17 in neuronal injury and microglial activation in oxygen-glucose deprivation/recovery (OGD/R)-, $\mathrm{LTD}_{4^{-}}$, or UDP-induced injury in vitro. The present study aimed to address the following questions: Determine the spatiotemporal profiles and localization of GPR17 in different cell cultures in vitro; assess whether GPR17 is correlated with neuronal injury and microglial activation following ischemia-like injury in vitro; and explore the mechanism by which GPR17 may regulate ischemia-like injury.

\section{Materials and methods}

Cell culture and $O G D / R$. Primary neurons were obtained from cerebral cortices of neonatal SD rats (Experimental Animal Center, Zhejiang Academy of Medicine Sciences, Hangzhou, China), as described in previous studies $(5,24)$. Briefly, cerebral cortices were dissected and digested with $0.25 \%$ EDTA-free trypsin (Gibco; Thermo Fisher Scientific, Inc., Waltham, MA, USA) at $37^{\circ} \mathrm{C}$ for $10 \mathrm{~min}$. Then, the dissociated cells were immediately seeded onto poly-L-lysine (Sigma-Aldrich; Merck KGaA, Darmstadt, Germany) pre-coated flasks, and incubated in high glucose DMEM (Gibco; Thermo Fisher Scientific, Inc.) containing $10 \%$ fetal bovine serum (FBS; Hangzhou Sijiqing Biotechnology Co., Hangzhou, China), 10\% horse serum (Gibco; Thermo Fisher Scientific, Inc.), 2 mM glutamine, $100 \mu \mathrm{g} / \mathrm{ml}$ streptomycin, and $100 \mathrm{units} / \mathrm{ml}$ penicillin (Sigma-Aldrich; Merck KGaA) for $24 \mathrm{~h}$ under normal culture conditions. After $24 \mathrm{~h}$ of culture, the medium was changed to high glucose DMEM containing 5\% horse serum, $2 \mathrm{mM}$ glutamine, 100 units $/ \mathrm{ml}$ penicillin, $100 \mu \mathrm{g} / \mathrm{ml}$ streptomycin, $0.04 \%$ B27, and $0.01 \%$ N2 (Gibco; Thermo Fisher Scientific, Inc.). The day of plating was recorded as DIV 0 in vitro. On DIV 3, $10 \mu \mathrm{M}$ cytosine arabinoside (Sigma-Aldrich; Merck $\mathrm{KGaA}$ ) was added for $24 \mathrm{~h}$ to inhibit the growth of glial cells in cultured neurons; the medium was refreshed every 3 days. On DIV $10,>95 \%$ of the cultured cells were neurons, as determined by immunofluorescent staining for microtubule-associated protein-2 (MAP-2; Millipore, Billerica, MA, USA).

For the neuron-glial mixed cultures of cortical cells, similar procedures were used, except for cytosine arabinoside supplementation, as previously described (25). On DIV 10 , $28 \%$ of the cultured cells were neurons, $7 \%$ were microglia and most of the remaining cells were astrocytes, as assessed by immunofluorescent staining for anti-MAP-2 (1:200; cat. no. AB5622; Millipore), anti-glial fibrillary acidic protein (GFAP; 1:800; cat. no. MAB3402; Millipore), and anti-ionized calcium-binding adapter molecule 1 (Iba1; 1:1,000; cat. no. 019-19741; Wako Pure Chemical Industries, Ltd., Osaka, Japan) antibodies.

Primary astrocytes were prepared from cerebral cortices of neonatal SD rats, as previously described $(4,26)$. Briefly, cerebral cortices were dissected and digested with $0.25 \%$ trypsin for $15 \mathrm{~min}$. Then, the dissociated cells were immediately plated onto poly-L-lysine pre-coated flasks, and incubated in high glucose DMEM containing 10\% FBS, 2 mM glutamine, $100 \mathrm{U} / \mathrm{ml}$ penicillin and $100 \mu \mathrm{g} / \mathrm{ml}$ streptomycin under normal culture conditions. Thereafter, the medium was refreshed every 3 days. On DIV 14, confluent cultures were shaken overnight at $250 \mathrm{rpm}$ at $37^{\circ} \mathrm{C}$ to reduce microglial contamination. Subsequently, adherent cells were digested with $0.25 \%$ trypsin and replated in the growth medium. More than $95 \%$ of the cultured cells were astrocytes, as assessed by immunofluorescent staining for GFAP.

Primary microglia were prepared from cerebral cortices of neonatal SD rats, as described previously $(5,27)$. Briefly, cerebral cortices were digested with $0.25 \%$ trypsin for $10 \mathrm{~min}$; then, the dissociated cells were immediately plated onto poly-L-lysine pre-coated flasks in antibiotics-free minimum Eagle's medium (Gibco; Thermo Fisher Scientific, Inc.) containing $10 \% \mathrm{FBS}$ at $37^{\circ} \mathrm{C}$. On DIV 10, the microglia cells were harvested by shaking for $30 \mathrm{~min}$ at $250 \mathrm{rpm}$. The cells were centrifuged and reseeded in the growth medium. More than $95 \%$ of the cultured cells were microglia, as determined by immunofluorescent staining for the microglial marker Iba-1.

To evaluate the effect of the microglial conditioned medium on primary neurons, cell supernatants were collected from the microglia pretreated with or without OGD/R as well as small interfering (si)RNA (5). The conditioned media was collected and centrifuged to remove cell debris. The primary neurons were incubated with the conditioned medium from the above microglia for $24 \mathrm{~h}$. Neuronal death was assessed subsequently.

For OGD $(4,5,28)$, cells were rinsed twice and cultured in Earle's solution without glucose. Then, the cells were incubated in an anaerobic chamber containing $95 \% \mathrm{~N}_{2}$ and $5 \% \mathrm{CO}_{2}$ at $37^{\circ} \mathrm{C}$ for $1 \mathrm{~h}$. Control cells were incubated in Earle's solution containing $25 \mathrm{mM}$ glucose under normal culture conditions for $1 \mathrm{~h}$. Following OGD, the cells were returned to the common incubator and cultured in regular medium to allow recovery.

The nonselective GPR17 agonists leukotriene D4 (LTD4) and uridine 5'-diphosphate (UDP; both Sigma-Aldrich; Merck $\mathrm{KGaA}$ ) were utilized for receptor activation. $\mathrm{LTD}_{4}$ or UDP was added to the medium at final concentrations of $0.1-1,000 \mathrm{nM}$ and $0.1-1,000 \mu \mathrm{M}$ for $24 \mathrm{~h}$, respectively.

All animal experiments were performed in accordance with the National Institutes of Health Guide for Care and Use of Laboratory Animals. The experimental protocols were approved by the Ethics Committee of Laboratory Animal Care and Welfare, School of Medicine, Zhejiang University (Hangzhou, China). 
GPR17 silencing by RNA interference. In the present study, siRNA was used to effectively downregulate GPR17 protein levels, for lack of a selective GPR17 antagonist. The specifically designed target sequence for rat GPR17 was CCG TAT AGA GAA GCA CCT CAA (GenePharma Co., Ltd., Shanghai, China) $(17,29)$. To rule out possible non-specific effects, a non-effective sequence (sequence, CCU ACG CCA CCA AUU UCG UTT) was used as negative control (NC). Transfection of siRNA duplexes was performed according to the manufacturer's instructions. Briefly, $24 \mathrm{~h}$ prior to transfection, the medium was changed to appropriate antibiotic-free medium containing $10 \%$ FBS. Then, GPR17 siRNA or NC siRNA was transiently transfected with Lipofectamine 2000 (Invitrogen; Thermo Fisher Scientific, Inc.) at a final concentration of $100 \mathrm{nM}$ for $6 \mathrm{~h}$; the cells were cultured for an additional $48 \mathrm{~h}$ prior to exposure to OGD/R, UDP or $\mathrm{LTD}_{4}$.

$M T T$ and lactate dehydrogenase (LDH) release assays. Following treatments, cell viability was evaluated by the MTT assay. Briefly, MTT (Sigma-Aldrich; Merck KGaA) was added to each well at a final concentration of $0.5 \mathrm{mg} / \mathrm{ml}$ and incubated for $4 \mathrm{~h}$ at $37^{\circ} \mathrm{C}$. After careful removal of the medium, $100 \mu$ 1 DMSO (Sigma-Aldrich; Merck KGaA) was added for 10 min with shaking. Finally, absorbance was measured at $490 \mathrm{~nm}$ on a microplate reader (Elx800; BioTek Instruments, Inc., Winooski, VT, USA). LDH released into the medium was quantified with a LDH detection kit (Jiancheng Co., Ltd., Nanjing, China), according to the manufacturer's instructions. LDH was detected at $450 \mathrm{~nm}$ on a microplate reader. Data were expressed as $\%$ of controls.

Immunofluorescence. Cells on cover slips in 24-well plates were fixed in cold methanol for $5 \mathrm{~min}$, followed by reaction with $10 \%$ normal goat serum (Zhongshan Biotechnology Co., Beijing, China) for $2 \mathrm{~h}$ to block non-specific IgG binding. Then, the cells were incubated with anti-MAP-2 (1:200; cat. no. AB5622; Millipore) anti-NeuN (1:500; cat. no. MAB377; Millipore), anti-GFAP (1:800; cat. no. MAB3402; Millipore), anti-Iba1 (1:1,000; cat. no. 019-19741; Wako Pure Chemical Industries, Ltd.), and anti-GPR17 (1:500; lab made) primary antibodies at $4^{\circ} \mathrm{C}$ overnight. Following rinsing with PBS, the cells were incubated with $\mathrm{Cy} 3$ - or fluorescein isothiocyanate (FITC)-conjugated secondary antibody (1:200; cat. nos. $\mathrm{AP} 187 \mathrm{C}$ and AP124F; Millipore) for $2 \mathrm{~h}$ at room temperature. After mounting with anti-fade medium (Invitrogen; Thermo Fisher Scientific, Inc.), the stained cells were visualized by fluorescence microscopy (BX51; Olympus Corporation, Tokyo, Japan). Negative control cover slips were incubated with normal goat serum instead of primary antibody. The polyclonal rabbit antibody against rat GPR17 used in the immunofluorescence is specific as reported $(9,14,17,30)$.

Assessment of cell death. To quantify cell death, cells grown on cover slips in 24-well plates were stained with $10 \mu \mathrm{g} / \mathrm{ml}$ propidium iodide (PI; Sigma-Aldrich; Merck KGaA) for $10 \mathrm{~min}$. Following rinsing and fixation in cold methanol for 5 min, immunofluorescent staining was performed by incubation with antibodies against NeuN (neuron marker), GFAP (astrocyte marker), and Iba-1 (microglia marker), respectively. After mounting with anti-fade medium, images were captured by fluorescent microcopy. The $\%$ of cell death was determined as the number of red PI-positive cells to total cells (as measured by DAPI staining). An observer blinded to the experimental treatments counted the cells.

Immunoblotting. Total cellular protein extraction was performed according to the manufacturer's instructions (Kangcheng Co., Ltd., Shanghai, China). Briefly, the cells were washed with PBS and lysed in Cell and Tissue Protein Extraction buffer on ice for $30 \mathrm{~min}$. Then, the homogenate was collected, centrifuged at $12,000 \times \mathrm{g}$ for $30 \mathrm{~min}$ at $4^{\circ} \mathrm{C}$, and protein amounts were quantified by Coomassie brilliant blue staining. Equal amounts of protein $(60 \mu \mathrm{g})$ for each sample were separated by $10 \%$ SDS-PAGE and transferred onto nitrocellulose membranes. The membranes were incubated with either goat polyclonal antibody against GPR17 (1:200; cat. no. sc-74791; Santa Cruz Biotechnology, Inc., Dallas, TX, USA) or mouse monoclonal antibody against GAPDH, (1:5,000; cat. no. KC-5G4; Kangcheng Co., Ltd., Shanghai, China) overnight at $4^{\circ} \mathrm{C}$. Subsequently, the membranes were treated with IRDye- $800 \mathrm{CW}$ donkey anti-goat IgG (1:3,000; cat. no. 926-32214; LI-COR Biosciences, Lincoln, NE, USA) or IRDye-800CW goat anti-mouse IgG $(1: 5,000$; cat. no. 610-132-121; Rockland Immunochemicals, Inc., Gilbertsville, PA, USA) for $2 \mathrm{~h}$ at room temperature. Images were scanned on an Odyssey fluorescence system (LI-COR Biosciences). Optical densities of GPR17 (41 kDa) and GAPDH $(36 \mathrm{kDa})$ were quantitatively assessed by Quantity One software (Bio-Rad Laboratories, Inc., Hercules, CA, USA).

Microglial phagocytosis. To evaluate the phagocytic activity of primary microglia, $1 \mathrm{~mm}$-diameter fluorescent carboxylate-modified microspheres (Millipore) were added to the cells for $1 \mathrm{~h}$. Subsequently, the cells were rinsed in PBS, immediately digested and resuspended, and transferred to flow-cytometry tubes. Finally, fluorescence was detected in the FL-3 channel by flow cytometry (BD Biosciences, Franklin Lakes, NJ, USA).

To evaluate the phagocytosis of microglia in mixed cortical cells, cover slips were placed in 24-well plates prior to cell seeding. Fluorescent microspheres were added to the cells for $1 \mathrm{~h}$ following treatment. After fixation and rinsing with PBS, the cells were incubated with $10 \%$ normal goat serum for $2 \mathrm{~h}$ at room temperature. To label the microglia, cells were treated with primary rabbit polyclonal antibody targeting Iba- $1(1: 1,000)$ at $4^{\circ} \mathrm{C}$ overnight. Following two washes, the cells were incubated with FITC-conjugated secondary antibody for $2 \mathrm{~h}$ at room temperature. This was followed by mounting with anti-fade medium; phagocytosis and the levels of the microglial marker Iba-1 were captured by fluorescence microscopy. Image analysis $(31,32)$ was performed based on a semi-quantitative method using ImageJ software (version 1.61; National Institutes of Health, Bethesda, MD, USA).

Inflammatory cytokine release measurement. Release of inflammatory cytokines, including tumor necrosis factor- $\alpha$ (TNF- $\alpha$ ) and interleukin-1 $\beta$ (IL-1 $\beta$ ), was detected with TNF- $\alpha$ (cat. no. RTA00; R\&D Systems, Inc., Minneapolis, MN, USA) and IL-1 $\beta$ (cat. no. EK0393; Boster Co., Ltd., Wuhan, China) ELISA kits, respectively. In brief, cultured cell 

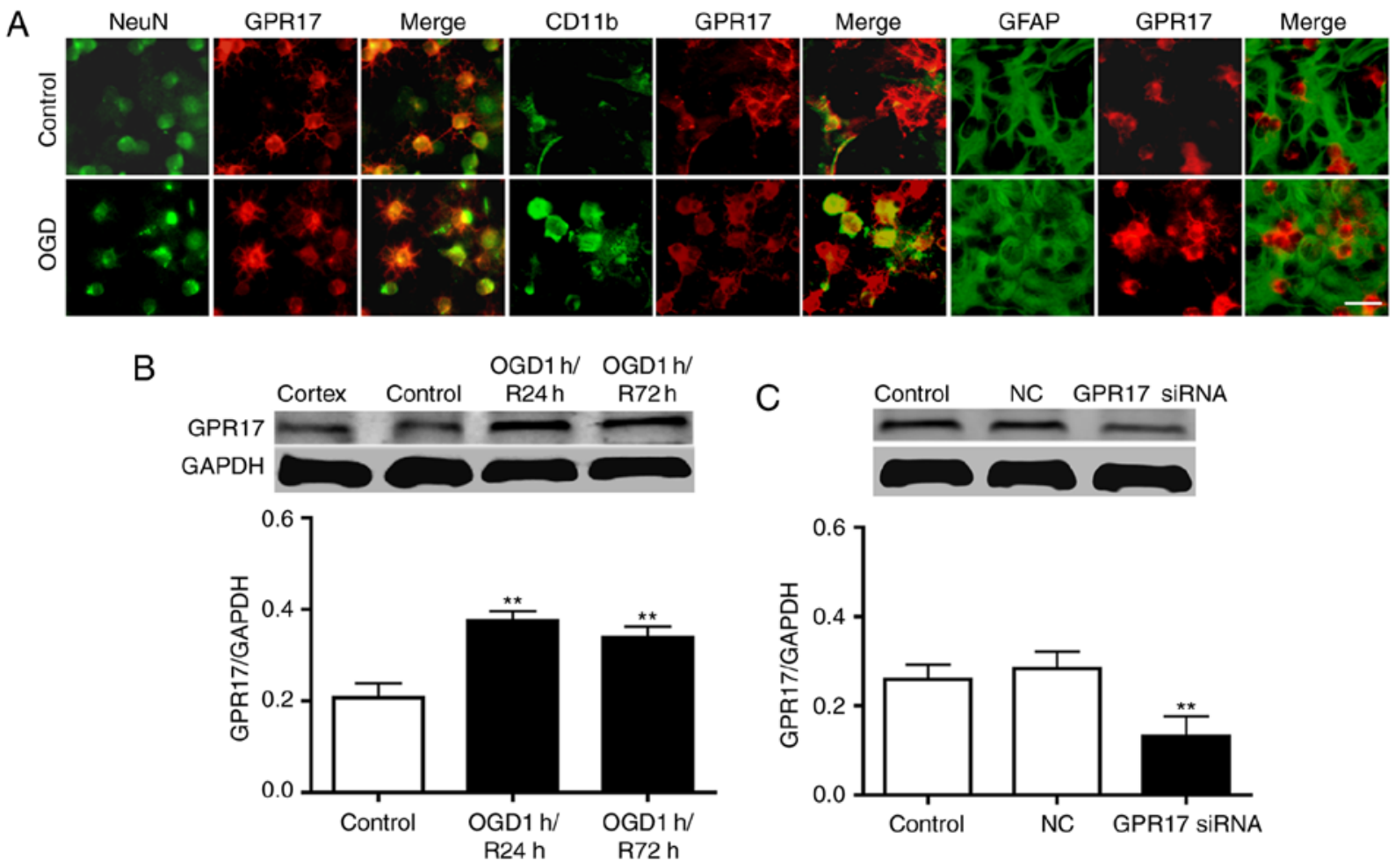

Figure 1. GPR17 distribution and expression in neuron-glial mixed cultures of cortical cells. (A) Representative images from microscopy analysis showing immunoreactivity to anti-GPR17 antibody (red) and the specific markers NeuN (neurons), CD11b (microglia) and GFAP (astrocytes) in neuron-glial mixed cultures of cortical cells, with or without ischemia-like injury. Ischemia-like injury was achieved by OGD for $1 \mathrm{~h}$ and recovery for $24 \mathrm{~h}$. Scale bar, $50 \mu \mathrm{m}$. (B) Western blotting revealed that GPR17 protein levels increased 24 and $72 \mathrm{~h}$ following recovery from OGD for $1 \mathrm{~h}$. (C) Reduced GPR17 expression in neuron-glial mixed cultures of cortical cells following treatment with specific GPR17 siRNA, but not a randomly designed NC siRNA. Data are presented as mean \pm standard error of the mean $(n=5) .{ }^{* *} \mathrm{P}<0.01$ compared with control. GPR17, G protein-coupled receptor 17 ; GFAP, glial fibrillary acidic protein; OGD, oxygen-glucose deprivation; si, small interfering; NC, negative control; R, recovery.

supernatants were collected following treatment, and cleared by centrifugation (670 x g for $10 \mathrm{~min})$. ELISA was performed according to the manufacturers' instructions. Optical densities were measured at $450 \mathrm{~nm}$ within $30 \mathrm{~min}$, and the amounts of released cytokine were derived from standard curves.

Statistical analysis. Data are mean \pm standard error of the mean. Groups were compared by one-way analysis of variance, followed by Dunnett's post hoc test, using SPSS 10.0 for Windows (SPSS, Inc., Chicago, IL, USA). P $<0.05$ was considered to indicate a statistically significant difference.

\section{Results}

Expression patterns of GPR17 in primary cortical cells. To determine the localization of GPR17 in vitro, double immunostaining was performed to assess the colocalization of GPR17 with the specific markers NeuN (neurons), GFAP (astrocytes), and CD11b (microglia) in neuron-glial mixed cultures with or without OGD/R. In control cells, most GPR17 expression was observed in NeuN-positive neurons, with low amounts in CD11b-positive microglia (Fig. 1A). Following OGD/R, GPR17 immunoreactivity was increased and evenly localized in NeuN-positive neurons and CD11b-positive microglia (Fig. 1A). By contrast, no colocalization was observed in GFAP-positive astrocytes, with or without OGD/R (Fig. 1A). Consistent with the immunostaining results, western blot analysis demonstrated that the GPR17 protein expression levels were significantly increased following exposure to OGD for $1 \mathrm{~h}$ and recovery for 24 or $72 \mathrm{~h}$ (Fig. 1B).

Effects of GPR17 knockdown on OGD/R-induced injury in neuron-glial mixed cultures of cortical cells. To further confirm the role of GPR17 in vitro, ischemia-like injury was assessed in neuron-glial mixed cultures, a cellular environment simulating the intact brain. Western blot analysis indicated that GPR17 siRNA treatment successfully downregulated GPR17 at the protein level, not only in neuron-glial mixed cultures (Fig. 1C), but also in primarily cultured neurons and microglia (data not shown); these findings were also verified by immunostaining (data not shown), indicating consistent GPR17 knockdown.

The results demonstrated that OGD for $1 \mathrm{~h}$ followed by recovery for $24 \mathrm{~h}$ resulted in decreased cell viability and increased LDH release in neuron-glial mixed cultures; these effects were attenuated by GPR17 siRNA (Fig. 2C and D). Thus, GPR17 knockdown exhibited remarkable protective effects on OGD/R-induced ischemic injury.

$\mathrm{PI} /$ Hoechst 33342 staining demonstrated that OGD for $1 \mathrm{~h}$ followed by recovery for $24 \mathrm{~h}(\mathrm{OGD} / \mathrm{R})$ induced obvious cell death (such as necrosis and pyroptosis) in neuron-glial mixed cultures, but apoptosis in very low levels (Fig. 2A). Next, cell death was assessed by double immunostaining with PI to characterize cell injury. In neuron-glial mixed cultures, OGD/R induced remarkable cell death in neurons and lower levels in Iba-1-positive microglia, with nearly no PI/GFAP-double 
A

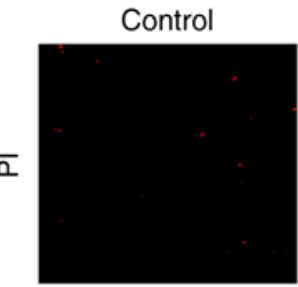

B
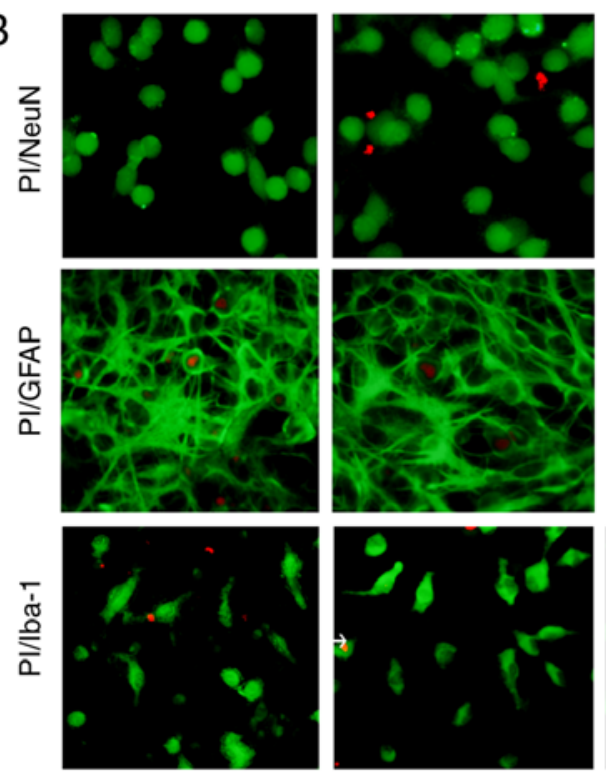
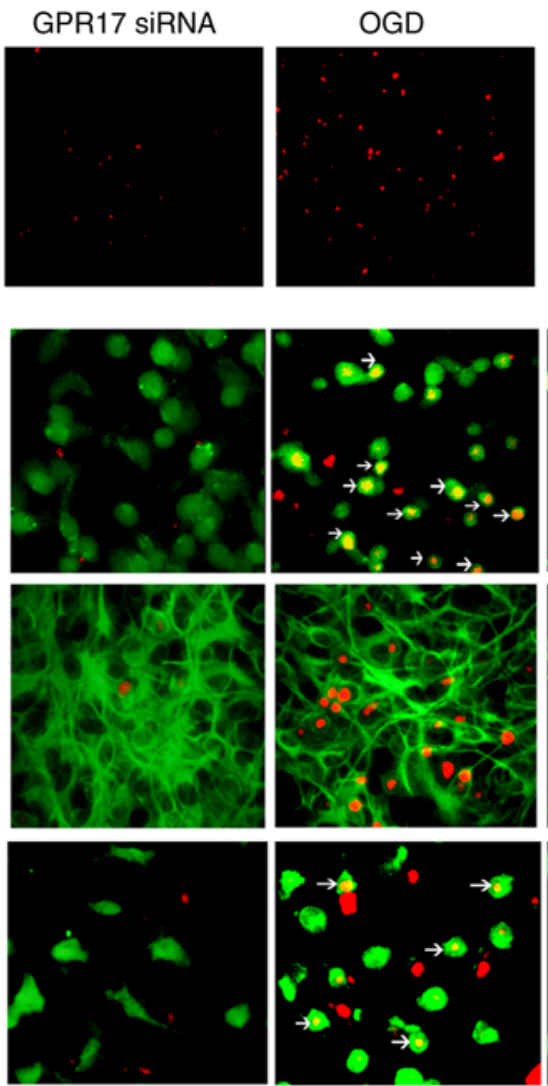

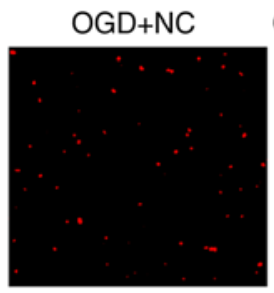

OGD+GPR17 siRNA
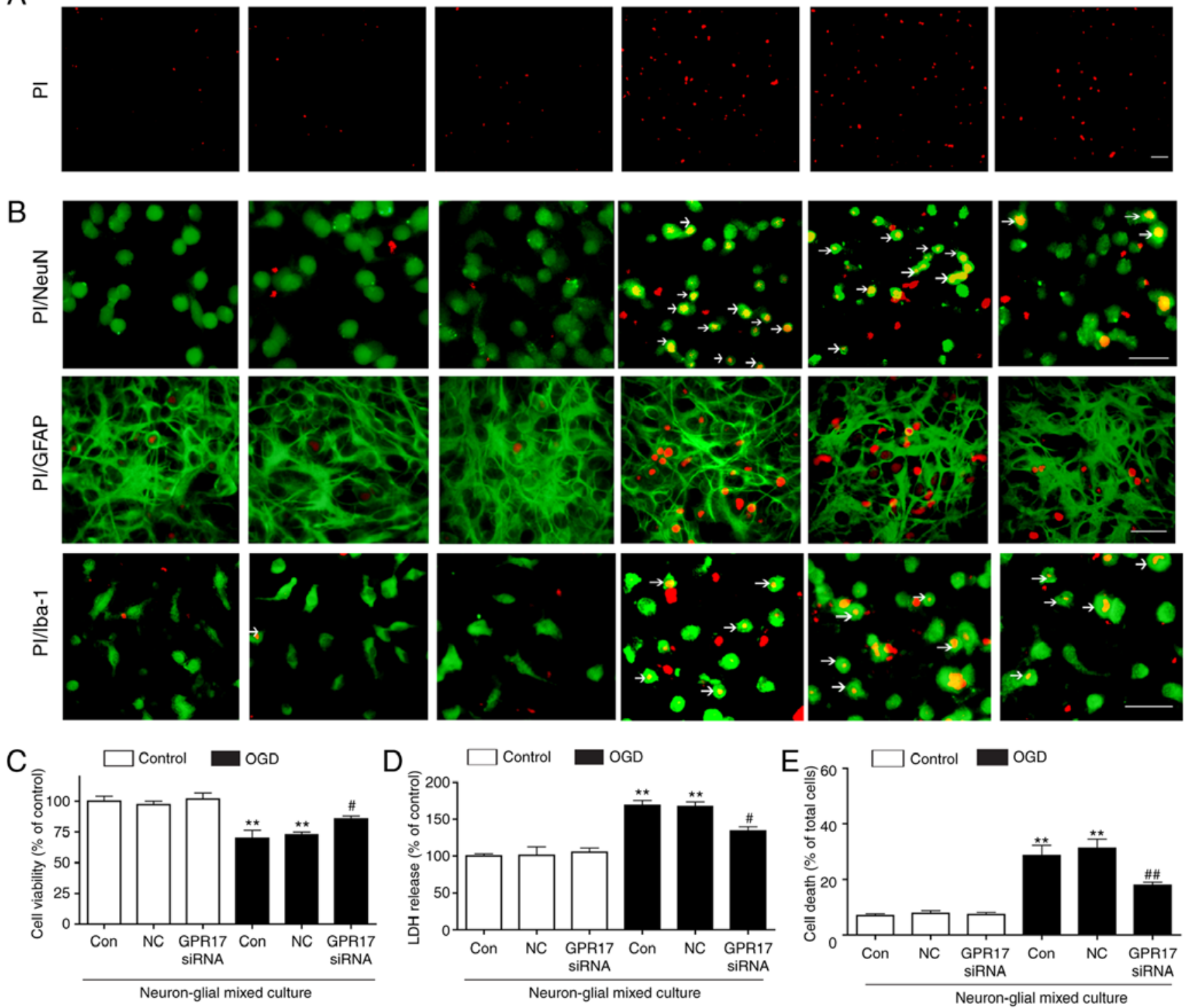

Figure 2. GPR17 mediates OGD/R-induced cell injury in neuron-glial mixed cultures of cortical cells. (A) Cell death in the neuron-glial mixed cultures was determined by PI staining (red). (B) Representative images from microscopy analysis showing co-staining of PI (red) with the specific markers NeuN, GFAP, or Iba-1 (green), with or without OGD/R. Arrows indicated the co-staining of PI with the specifc marker (NeuN, GFAP, or Iba-1). (C) Cell viability was measured by MTT assay. (D) LDH release. (E) Quantification of PI staining as a measure of cell death. Data are presented as mean \pm standard error of the mean ( $=6-8)$. ${ }^{* *} \mathrm{P}<0.01$ compared with control; ${ }^{\# P}<0.05$ and ${ }^{\# \#} \mathrm{P}<0.01$ compared with the OGD alone. Scale bar, $50 \mu \mathrm{m}$. GPR17, G protein-coupled receptor 17; OGD/R, oxygen-glucose deprivation/recovery; PI, propidium iodide; GFAP, glial fibrillary acidic protein; LDH, lactate dehydrogenase; si, small interfering; NC, negative control.

positive astrocytes observed (Fig. 2B). Application of GPR17 siRNA significantly ameliorated OGD/R-induced increase of cell death (Fig. 2A and E); the negative control siRNA had no such effects. Therefore, GPR17 knockdown exerted obvious protective effects on OGD/R-induced ischemic cell damage in neuron-glial mixed cultures. These results indicated that GPR17 might mediate OGD/R-induced ischemic injury in neuron-glial mixed cultures.

Effects of GPR17 knockdown on agonist-induced cell injury in neuron-glial mixed cultures of cortical cells. Next, the present study assessed whether GPR17 agonists had a direct injury effect in neuron-glial mixed cultures, similar to OGD/R-induced ischemic injury. Following treatment with UDP $(10-1,000 \mu \mathrm{M})$ or $\mathrm{LTD}_{4}(10-1,000 \mathrm{nM})$, it was observed that GPR17 agonists decreased cell viability and increased
$\mathrm{LDH}$ release in a dose and time-dependent manner (data not shown). Moderate amounts of $\mathrm{LTD}_{4}(100 \mathrm{nM})$ or UDP $(100 \mu \mathrm{M})$ for $48 \mathrm{~h}$ were selected as the optimal conditions in subsequent experiments. Notable, GPR17 siRNA silencing, and not negative control siRNA, significantly alleviated the reduced cell viability and enhanced $L D H$ release induced by UDP (Fig. 3A and B) or $\mathrm{LTD}_{4}$ (Fig. 3D and E).

Subsequently, the present study evaluated whether GPR17 was involved in $\mathrm{LTD}_{4}$ or UDP-induced cell death. The results demonstrated that $\mathrm{LTD}_{4}$ or UDP induced significant cell death in neuron-glial mixed cultures, and application of GPR17 siRNA significantly ameliorated the $\mathrm{LTD}_{4}$ or UDP-induced cell death (Fig. 3C and F); by contrast, negative control siRNA did not affect cell death. These findings indicated that GPR17 knockdown could alleviate LTD $_{4}$ or UDP-induced ischemia-like injury in neuron-glial mixed cultures. 
A
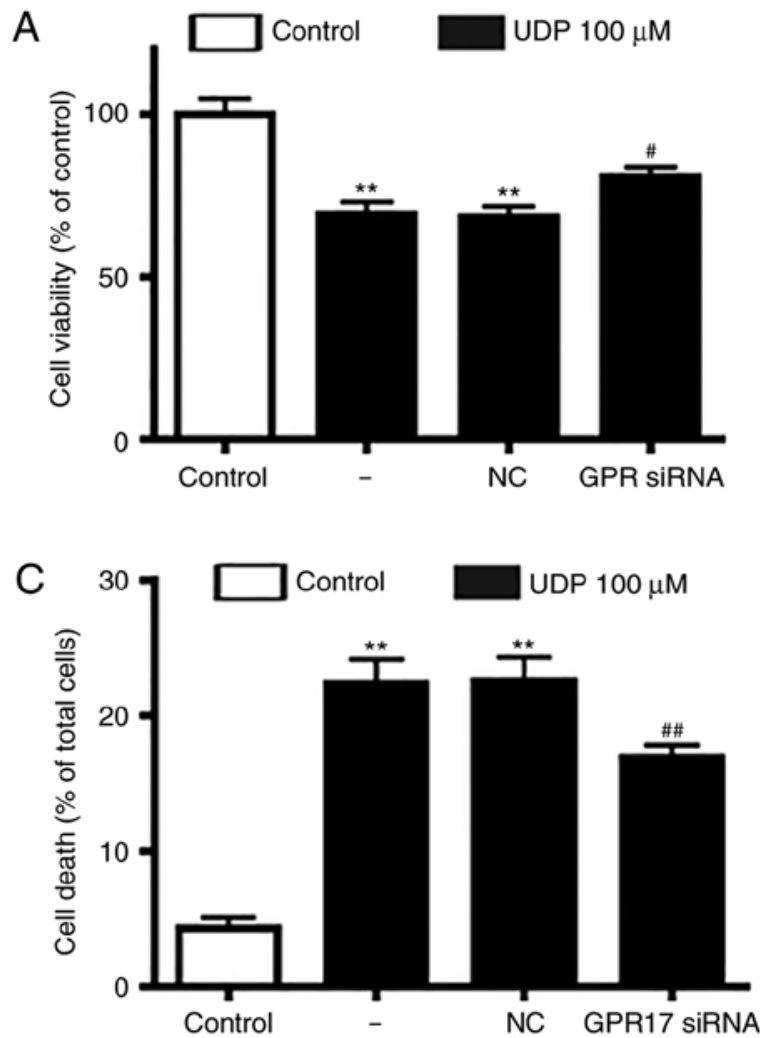

E

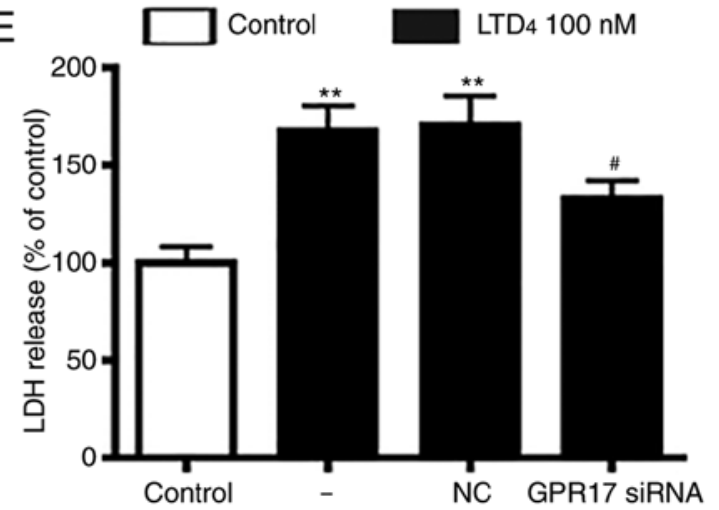

B

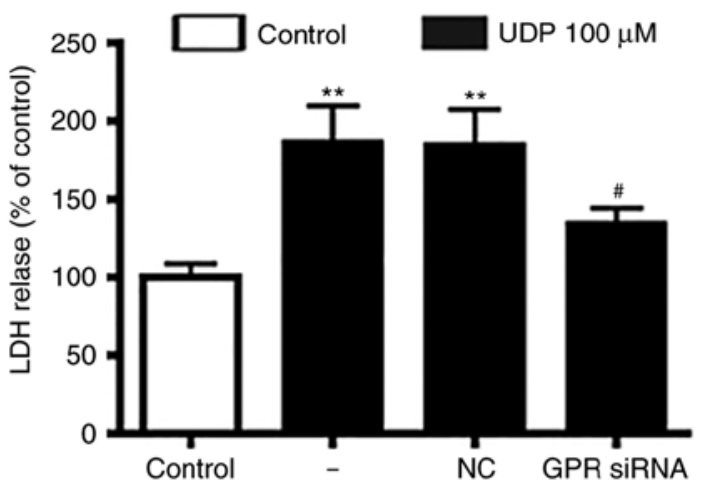

D

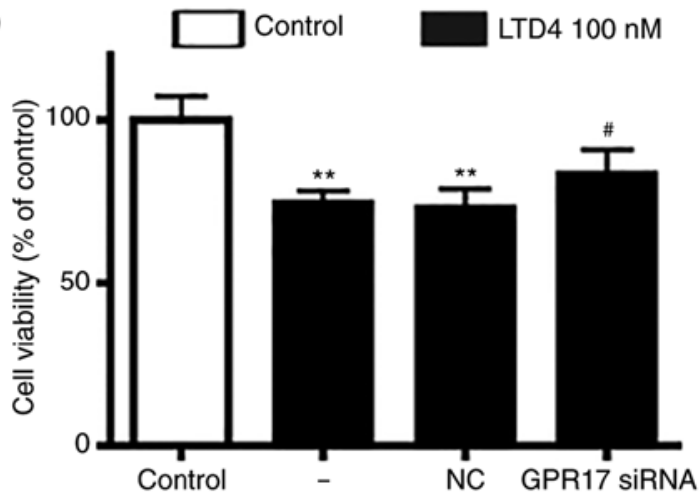

F

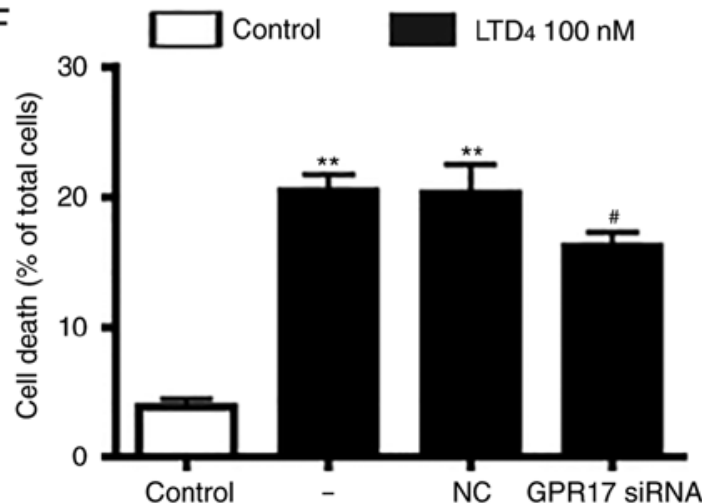

Figure 3. Agonist-induced cell injury and effects of GPR17 on neuron-glial mixed cultures of cortical cells. Cell viability (by MTT assay), LDH release and cell death (by PI staining) were measured in primary neuron-glial mixed cultures following exposure to (A-C) UDP (100 $\mu \mathrm{M}$ ), or (D-F) LTD 4 (100 $\mathrm{nM}$ ) for $48 \mathrm{~h}$. Data are presented as mean \pm standard error of the mean $(\mathrm{n}=6-8)$. ${ }^{* *} \mathrm{P}<0.01$ compared with control; ${ }^{\#} \mathrm{P}<0.05$ and ${ }^{\# \#} \mathrm{P}<0.01$ compared with $\mathrm{UDP}$ or $\mathrm{LTD}$ alone. GPR17, G protein-coupled receptor 17; LDH, lactate dehydrogenase; PI, propidium iodide; UDP, uridine 5'-diphosphate; LTD 4 , leukotriene $\mathrm{D}_{4}$; si, $_{\text {, small }}$ interfering; $\mathrm{NC}$, negative control.

Effects of GPR17 RNA knockdown on OGD/R-induced microglial activation in neuron-glial mixed cultures of cortical cells. To clarify the regulation of microglial activation by GPR17, changes of microglial morphology, phagocytosis, and release of proinflammatory cytokines, as indicators of microglial activation in neuron-glial mixed cultures, were assessed. In control conditions, ramified Iba-1-positive microglia persisted at a relatively high ratio, whereas microglia with activated appearance (rounded or amoeboid macrophage-like) progressively increased following exposure to OGD/R. GPR17 siRNA treatment ameliorated the ratio of activated microglia, whereas the negative control siRNA had no effect (Fig. 4A and C). In addition, OGD/R significantly enhanced microglial phagocytosis, an effect significantly inhibited by GPR17 siRNA as assessed microscopically (Fig. 4B and D).
Furthermore, OGD/R promoted the release of inflammatory cytokines TNF- $\alpha$ (Fig. 4E) and IL-1 $\beta$ (Fig. 4F), and this effect was significantly inhibited by GPR17 siRNA (Fig. 4E and F). These findings indicated that GPR17 mediated microglial activation, including morphological changes, phagocytosis and inflammatory cytokine release in neuron-glial mixed cultures.

Effects of GPR17 knockdown on OGD/R-induced cell injury in primary neurons and astrocytes. To explore the detailed mechanisms involved in the protective effects of GPR17 knockdown against OGD/R-induced ischemia-like injury, primary neurons and astrocytes were assessed, respectively.

In primary astrocyte cultures, GPR17 was not even expressed following exposure to OGD/R (data not shown). 
A
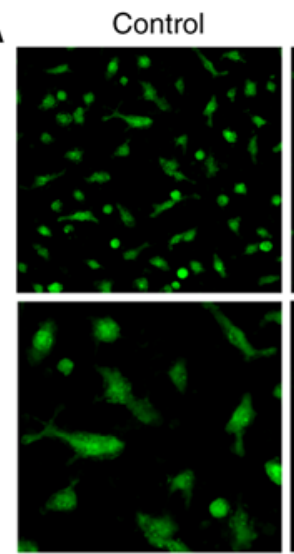

B

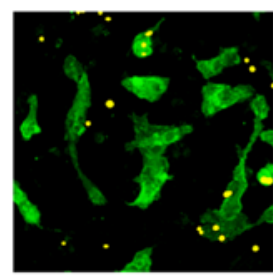

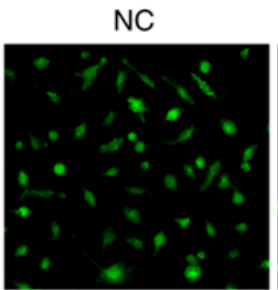
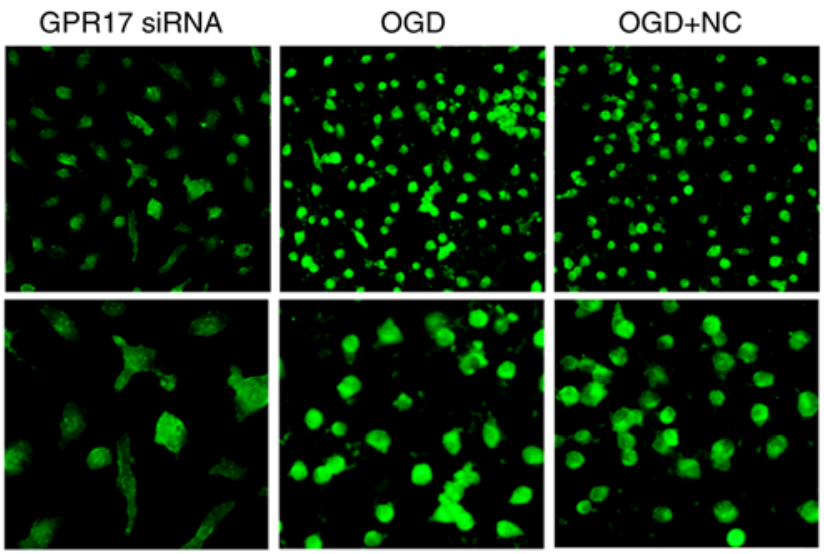

OGD+GPR17 siRNA
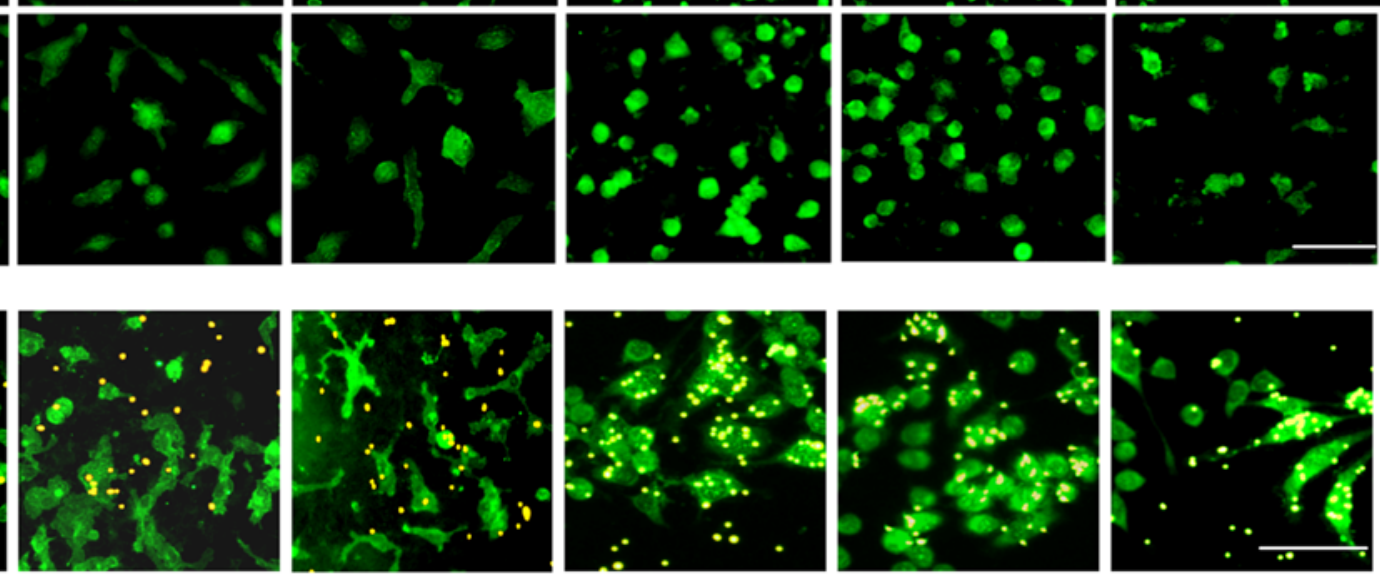

C

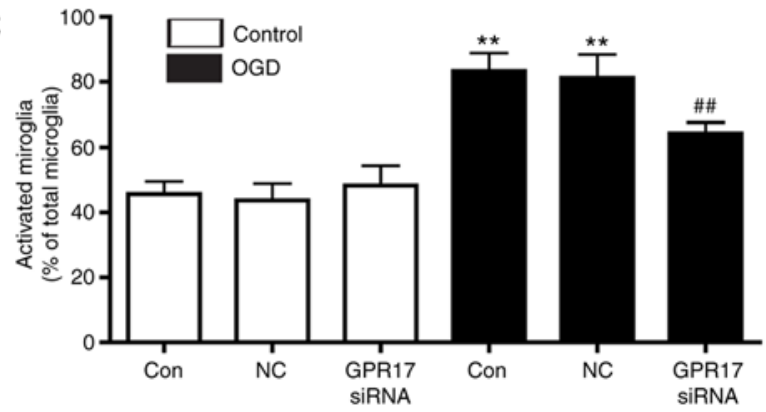

E

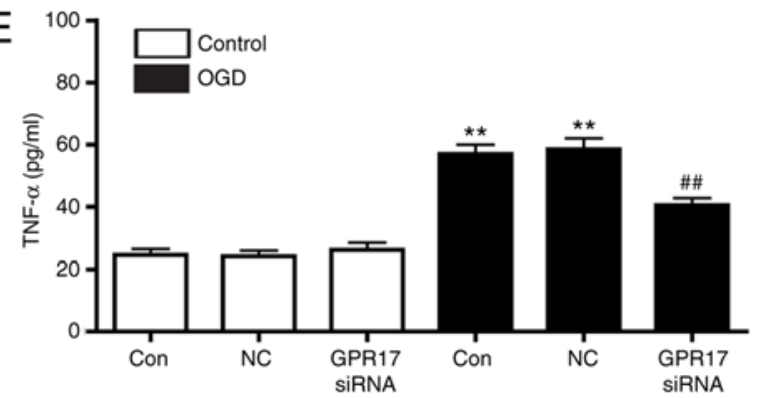

D $\left.{ }^{250}\right] \square$ Control

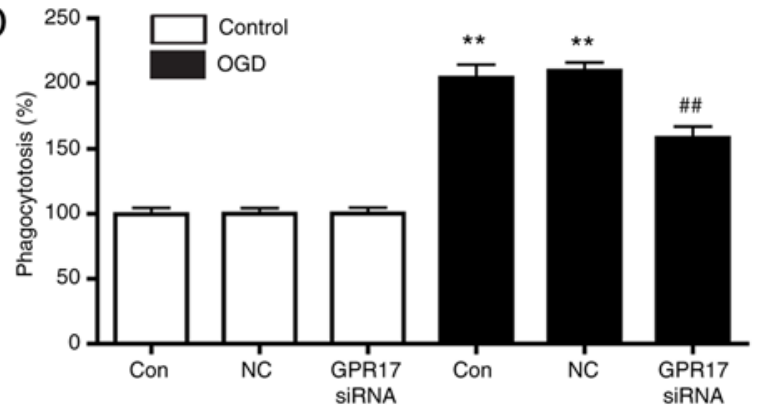

F

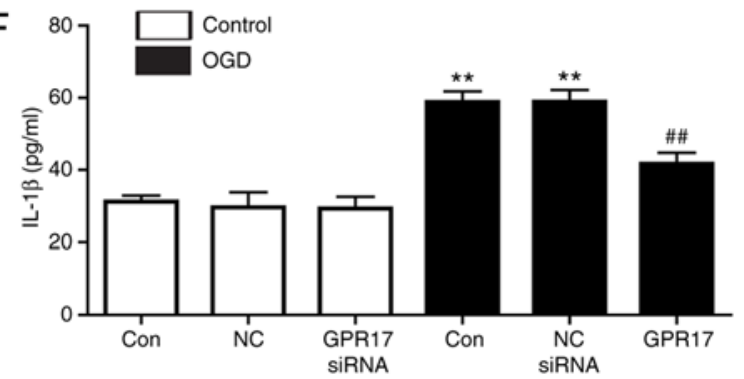

Figure 4. GPR17 mediates OGD/R-induced microglial activation, phagocytosis, and proinflammatory cytokine release in the primary neuron-glial mixed culture of cortical cells. (A) Representative images from microscopy analysis of microglia morphology with Iba-1 staining. (B) Representative images from microscopy analysis showing phagocytic activity of microglia after different treatments. Scale bar, $50 \mu \mathrm{m}$. (C) Quantification of staining in panel A. (D) Quantification of staining in panel B. (E) Levels of TNF- $\alpha$ and (F) IL- $1 \beta$ in the culture medium were evaluated by ELISA. Data are presented as mean \pm standard error of the mean $(n=6) .{ }^{* *} \mathrm{P}<0.01$ compared with control; ${ }^{\# \#} \mathrm{P}<0.01$ compared with OGD/R alone. GPR17, G protein-coupled receptor 17 ; OGD/R, oxygen-glucose deprivation/recovery; TNF- $\alpha$, tumor necrosis factor- $\alpha$; IL-1 $\beta$, interleukin-1 $\beta$; si, small interfering; NC, negative control.

Consistent with previous reports (26), OGD for $1 \mathrm{~h}$ and recovery for $24 \mathrm{~h}$ (simulating moderate injury) did not induce significant changes in cell viability and LDH release in primary astrocyte cultures, and GPR17 siRNA did not affect astrocyte viability, $\mathrm{LDH}$ release and cell damage following exposure to OGD/R (Fig. 5A and C-E). GPR17 or OGD/R did not induce detectable necrosis in primary astrocyte cultures (Fig. 5E). In primary neuron cultures, OGD for $1 \mathrm{~h}$ and recovery for $24 \mathrm{~h}$ resulted in decreased neuronal viability, and increased LDH release; however, in contrast to the findings from neuron-glial mixed cultures, the OGD/R-induced injury was not attenuated by GPR17 siRNA (Fig. 5B, F and G). In addition, OGD/R induced remarkable neuronal death in primary neuron cultures, however, increased neuronal injury was not inhibited by GPR17 siRNA (Fig. 5B and H).

These findings indicated that the protective effects of GPR17 knockdown against OGD/R-induced ischemic neuronal injury were not directly on neurons, at least following moderate OGD/R treatment, which cannot explain the in vitro findings for neuron-glial mixed cultures, as well as in previous in vivo data $(9,14,17)$. Therefore, it is possible that GPR17 might regulate ischemic neuronal injury via interactions 

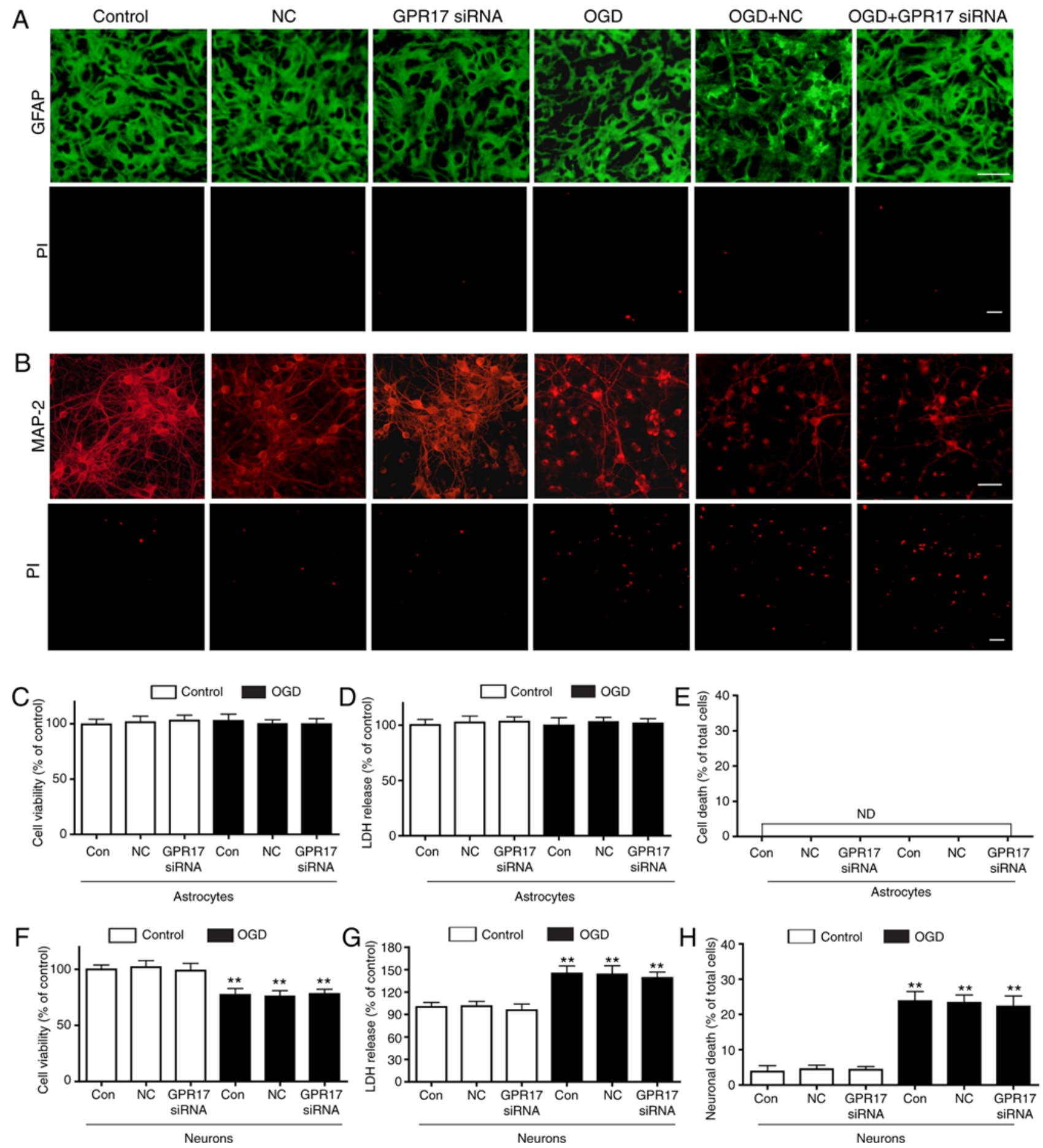

Figure 5. GPR17 is not involved in OGD/R-induced injury in primary neurons or astrocytes. Representative images from microscopy analysis showing the morphology of (A) astrocytes and (B) neurons immunostained with GFAP or MAP-2, respectively. Cell death was evaluated by PI staining. Scale bar, $50 \mu \mathrm{m}$. (C) Cell viability was detected by the MTT assay in astrocytes. (D) LDH release in astrocytes. (E) Quantification of cell death by PI staining in astrocytes. (F) Cell viability was detected by the MTT assay in neurons. (G) LDH release in neurons. (H) Quantification of cell death by PI staining in neurons. Data are presented as mean \pm standard error of the mean $(n=6-8){ }^{* *} \mathrm{P}<0.01$ compared with control. GPR17, G protein-coupled receptor 17; OGD/R, oxygen-glucose deprivation/recovery; GFAP, glial fibrillary acidic protein; MAP-2, microtubule-associated protein-2; PI, propidium iodide; LDH, lactate dehydrogenase; si, small interfering; $\mathrm{NC}$, negative control; $\mathrm{ND}$, not detectable.

between neurons and surrounding cells, such as astrocytes and microglia. However, the results demonstrated that astrocytes were not involved in the protective effects of GPR17 knckdown. Thus, it can be speculated that other cells in the mixed cortical cell cultures used in the present study, perhaps microglia, may be associated with the protective effects of GPR17 knockdown, which might eventually cause neuronal injury.
Effects of GPR17 knockdown on OGD/R-induced microglial activation in primary microglia cultures. To explore whether GPR17 directly regulates microglial activation, microglial phagocytosis and proinflammatory cytokine release were assessed in primary microglia cultures. Flow cytometry analysis demonstrated that OGD/R significantly enhanced microglial phagocytosis, and this effect was significantly 
A

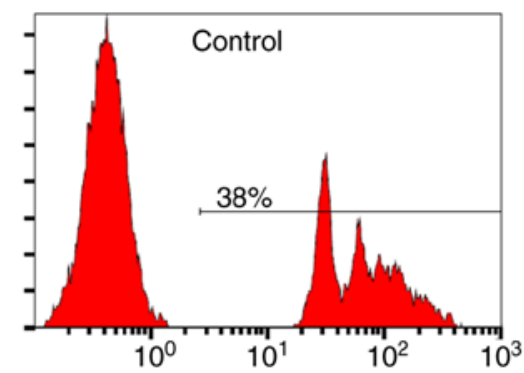

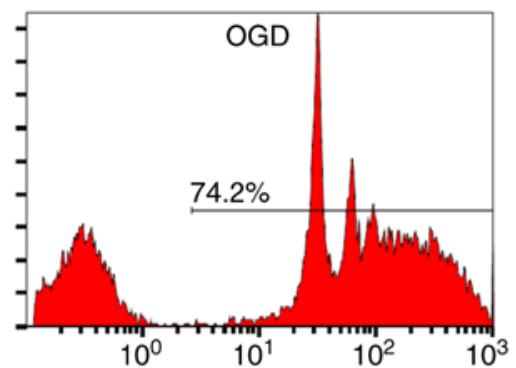
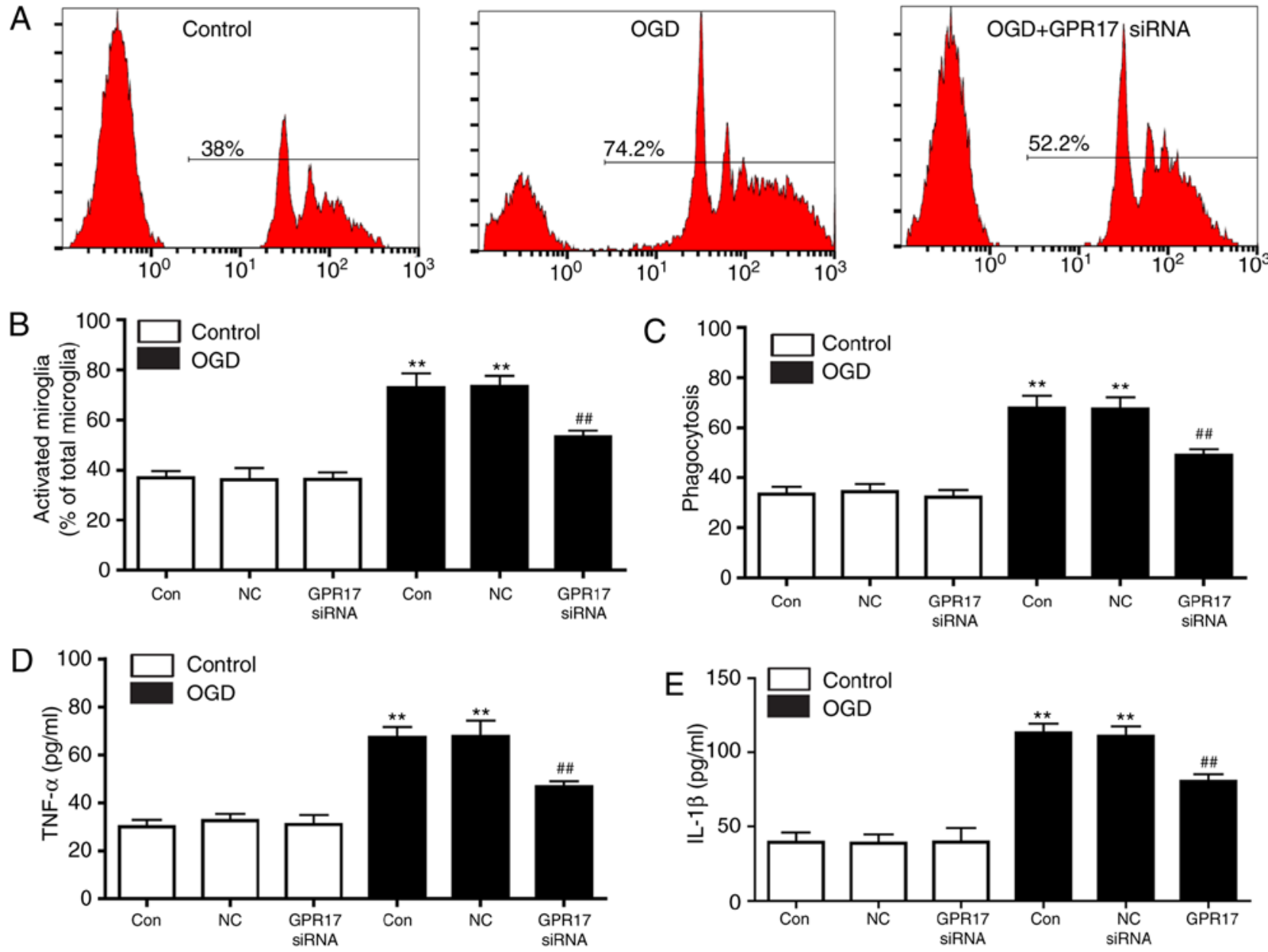

Figure 6. GPR17 mediates OGD/R-induced microglial activation, phagocytosis, and proinflammatory cytokine release in primary microglia. (A) Representative plots from flow cytometry analysis of microglial phagocytosis in primary microglia. (B) Quantification of morphological changes of microglia. (C) Quantification of flow cytometry results of panel A. (D) Levels of TNF- $\alpha$ and (E) IL-1 $\beta$ in the culture medium were detected by ELISA. Data are presented as mean \pm standard error of the mean $(\mathrm{n}=6) .{ }^{* *} \mathrm{P}<0.01$ compared with control; ${ }^{\# \prime} \mathrm{P}<0.01$ compared with OGD alone. GPR17, G protein-coupled receptor 17; OGD/R, oxygen-glucose deprivation/recovery; TNF- $\alpha$, tumor necrosis factor- $\alpha$; IL-1 $\beta$, interleukin- $1 \beta$; si, small interfering; NC, negative control.

suppressed by GPR17 siRNA (Fig. 6A and C). In addition, in control conditions, microglia with activated appearance (rounded or amoeboid macrophage-like) persisted at a relatively low ratio, whereas activated microglia progressively increased following exposure to OGD/R (Fig. 6B). GPR17 siRNA treatment ameliorated the ratio of activated microglial, whereas the negative control siRNA had no effect (Fig. 6B). Finally, OGD/R significantly increased the release of the proinflammatory cytokines TNF- $\alpha$ and IL- $1 \beta$, however, this effect was significantly inhibited by GPR17 siRNA (Fig. 6D and E). These findings indicated that GPR17 knockdown directly inhibited OGD/R-induced microglial activation in primary microglia cultures.

Effects of GPR17 knockdown on microglial-conditioned medium-induced neuronal injury. Finally, neuronal death was investigated in primary neurons induced by the conditioned medium from microglia, pretreated with or without OGD/R, as well as siRNA. As illustrated in Fig. 7, the conditioned medium pretreated with $\mathrm{OGD} / \mathrm{R}$ was able to induce neuronal death, and this effect was significantly inhibited by GPR17 siRNA pretreatment, while control siRNA had no effect (Fig. 7A and B).
Taken together, these findings suggested that GPR17 might directly mediate microglial activation, and this effect might be the primary way for regulating ischemic neuronal injury in mixed cultures of cortical cells. However, whether GPR17 regulates neuronal injury mediated by oligodendrocytes remains to be investigated.

\section{Discussion}

The present study confirmed and extended previous studies which reported that GPR17 upregulation is spatiotemporally correlated with neuronal injury and microglial activation in vivo. In addition, the present study provided further evidence that GPR17 knockdown attenuated ischemia-like neuronal injury only in neuron-glial mixed cultures of cortical cells, and not in primary neuron cultures, indicating the participation of glia cells in the process. In addition, GPR17 knockdown was demonstrated to suppress OGD/R-induced microglial activation, inducing phagocytosis and inflammatory cytokine release, in both neuron-glial mixed cortical cells and primary microglia. An important finding of the present study was that the conditioned medium from microglia pretreated with $\mathrm{OGD} / \mathrm{R}$ induced neuronal death, and the injury was 
A
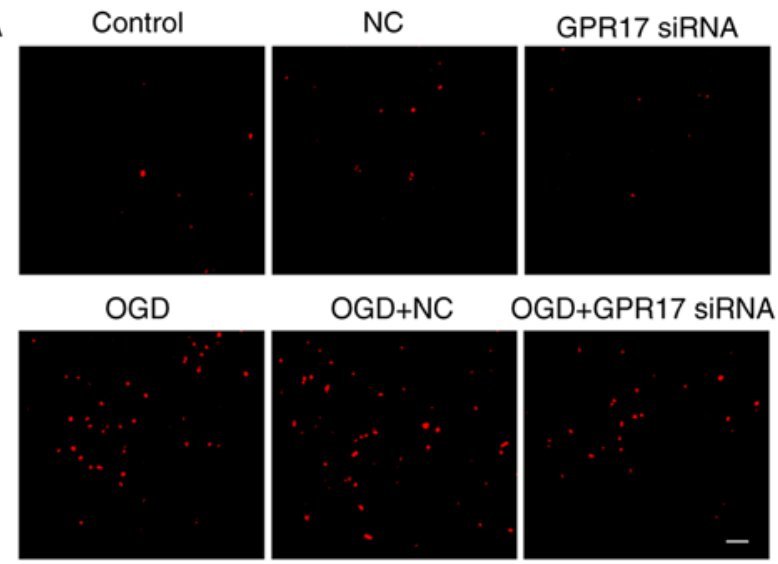

$\mathrm{OGD}+\mathrm{NC}$
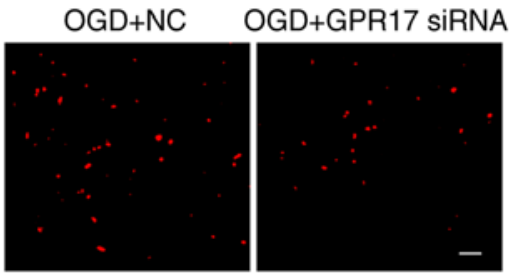

B

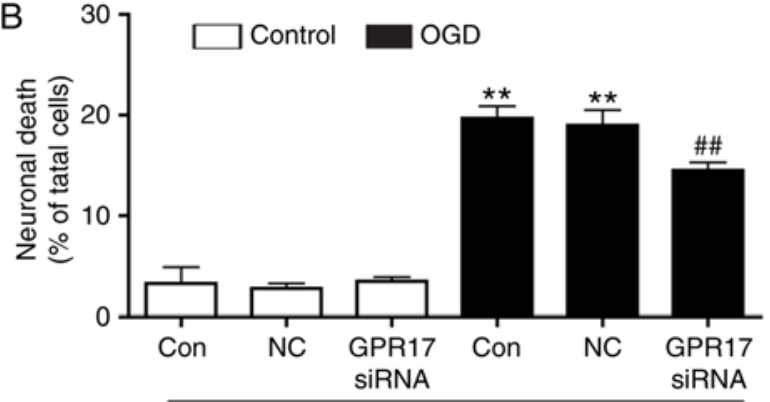

Neurons

Figure 7. GPR17 attenuates neuronal death induced by conditioned medium from microglia. Conditioned medium from microglia pretreated with or without OGD/R and siRNA was added into primary neuron cultures, and neuronal death was determined by PI staining. (A) Representative images from microscopy analysis. (B) Quantification of cell death. Data are presented as mean \pm standard error of the mean $(n=8)$. ${ }^{* *} \mathrm{P}<0.01$ compared with control ${ }^{\#} \mathrm{P}<0.01$ compared with the OGD alone. GPR17, G protein-coupled receptor 17; OGD/R, oxygen-glucose deprivation/recovery; PI, propidium iodide; si, small interfering; NC, negative control.

significantly inhibited by GPR17 siRNA pretreatment. To the best of our knowledge, this is the first description of functional effects of GPR17 in a native in vitro system mimicking the intact brain, as well as its role in ischemia-like microglial activation and neuronal injury in vitro.

The current results also confirmed that GPR17, activated by the agonists UDP and $\mathrm{LTD}_{4}$, enhanced LDH release and neuronal death, while reducing cell viability; these effects were attenuated by GPR17 knockdown in neuron-glial mixed cultures of cortical cells. These findings corroborated previous reports demonstrating that GPR 17 is phylogenetically linked to P2Y receptors for nucleotides and cysteinyl leukotriene receptors for CysLTs (8-10). However, with regard to the activation of GPR17 by nucleotides and cysteinyl leukotrienes, results from different laboratories remain controversial and require further study (33-38).

Currently, GPR17 has mainly been assessed for its roles in regulating OPC differentiation and myelination (19-22). Mounting evidence indicates that GPR17 represents one of the potential G protein-coupled receptor (GPCR) drug targets for demyelinating diseases, such as multiple sclerosis $(20,39,40)$. Previous studies reported that GPR17 also acts as a damage sensor in cerebral ischemic injury and spinal cord damage, and might serve important roles in the modulation of both ischemic neuronal injury and late remodeling/repair responses $(9,14,17,18)$. Following cerebral ischemia, GPR17 upregulation in neurons was reported to be associated with enhanced cell damage, especially within $24 \mathrm{~h}$ of reperfusion (microglia not markedly activated); GPR17 knockdown attenuated acute ischemic injury, strongly indicating its role in neuronal death in vivo $(9,14,17)$. While in the late phase (14-28 days) of focal cerebral ischemia, GPR17 level increase is mainly localized in microglia/microphages in the penumbra and ischemic core, its knockdown results in attenuated brain atrophy and post-ischemic microgliosis, indicating GPR17 involvement in the modulation of microglial activation $(14,17)$. However, whether ischemia-like neuronal injury and microglial activation in vitro are mediated by GPR 17 is poorly understood. A better understanding of the underlying mechanism is crucial for the development of effective therapeutics to ischemic stroke.

In the present study, neuron-glial mixed cultures were used to simulate the brain environment. Consistent with previous reports, $\sim 28 \%$ of cultured cells were neurons, $7 \%$ were microglia cells, and most of the remaining cells were astrocytes in the neuron-glial mixed cultures of cortical cells (25). Following moderate ischemia-like injury (OGD for $1 \mathrm{~h}$ and recovery for $24 \mathrm{~h}$ ), neuronal death and microglial activation were significantly increased, whereas astrocytes were not injured (nearly no necrosis or morphological changes). GPR17 immunoreactivity was observed in neurons and microglia, but not in astrocytes even after OGD/R. As demonstrated in the current study, GPR17 siRNA attenuated ischemic neuronal injury only in neuron-glial mixed cultured cells, and not in primary neuron cultures. Combined with in vivo findings $(9,14,17)$, it can be speculated that GPR17 on glial cells might directly mediate responses to uracil nucleotides and CysLTs, and secondarily induce ischemia-like neuronal injury. However, GPR17 siRNA did not affect astrocyte responses in both neuron-glial mixed cultured cells and primary astrocyte cultures. In addition, oligodendrocyte linkage persisted at very low levels in the neuron-glial mixed cultures of cortical cells $(5,25)$. Based on these findings, the present study was designed to further investigate the effect of microglial activation. Nevertheless, the present experiments did not remove all of the oligodendrocyte linkage in neuron-glial mixed cultured cells, and thus the possibility that oligodendrocyte linkage may have a regulatory role in the present study cannot be excluded.

Microglia constitute the first immune defense in the central nervous system, and have dual roles in physiological and pathological conditions $(41,42)$. Activated microglia can protect neurons against damage by phagocytosis of cellular debris, release of neurotrophic and anti-inflammatory mediators $(41,43)$. Nevertheless, uncontrolled or overactivated microglia can trigger neurotoxicity by releasing harmful substances, such as inflammatory cytokines, reactive oxygen species, and proteinases $(42,44)$. Thus, microglial activation and the subsequent inflammation-mediated neurotoxicity have crucial roles in the pathogenesis of neurodegenerative diseases, including cerebral ischemia.

In neuron-glial mixed cultures, GPR17 knockdown inhibited OGD/R-induced microglial activation, inducing phagocytosis and the release of inflammatory cytokines TNF- $\alpha$ and IL-1 $\beta$. Thereafter, primary microglia cultures were used to explore whether GPR17 could directly regulate microglial 
activation. The current findings revealed that GPR17 knockdown inhibited OGD/R-induced microglial activation by decreasing microglial phagocytosis, and inhibiting the release of TNF- $\alpha$ and IL-1 $\beta$. In line with these findings, colleagues have demonstrated that GPR17 RNA interference inhibited phagocytosis, suppressed the expression of iNOS and IL-1 $\beta$, following OGD/R in BV2 microglial cells (unpublished data). In addition, the microglial conditioned medium pretreated with OGD/R was able to induce neuronal death, which was significantly inhibited by GPR17 siRNA pretreatment. Combined with the present results, it can be speculated that GPR17 might directly mediate microglial activation and, in turn, cause neuronal injury in the mixed culture of cortical cells, although further direct evidence is required for confirmation.

The regulatory role of GPR17 in microglial activation in ischemic/inflammatory diseases might be a response to the changes of extracellular nucleotides and CysLTs. The above changes were supported by the following evidences: Neurons can release both adenine (such as ATP) and uracil (such as UTP and UDP) nucleotides into the extracellular space in response to ischemic/inflammatory damage $(45,46) ; 1321 \mathrm{~N} 1$ human astrocytoma cells release uracil nucleotides (UTP) and nucleotide sugar (UDP-glucose) in response to various stimuli $(47,48)$; excitatory kainic acid can increase extracellular UTP in vivo and in vitro (45). In addition, CysLT levels are significantly elevated at $3 \mathrm{~h}$, and persisted for $24 \mathrm{~h}$ in the ischemic brain $(49,50)$; OGD/R can increase CysLT release in cortical cells $(4,26)$. Under these conditions, GPR17 might operate as a sensor molecule regulating microglial activation.

The present study cannot exclude the possibility that GPR17 in oligodendrocyte linkage has a role in modulating neuronal injury and microglial activation, although oligodendrocytes existed in minute amounts in the present neuron-glial mixed cultures. Additionally, there is evidence of probable associations of GPR 17 with other G protein coupled receptors that serve important roles in allergic pulmonary inflammation and oligodendrocyte myelination processes $(8,51,52)$. CysLT 1 and CysLT $_{2}$ receptors exhibit expression and localization patterns similar to those of GPR17 in the brain, and are remarkably upregulated and localized in microglia during the late phases of ischemic brain $(53,54)$. The aforementioned evidence suggests that other regulatory mechanisms may be present in the role of GPR 17 in OGD/R-induced microglial activation and neuronal injury, and this requires further investigation.

In summary, the present study confirmed that GPR17 upregulation was associated with microglial activation and neuronal injury in vitro. GPR17 knockdown attenuated ischemia-like neuronal injury only in neuron-glial mixed cultures, and not directly in neurons or astrocytes. Additionally, GPR 17 knockdown attenuated OGD/R-induced microglial activation, inducing phagocytosis and inflammatory cytokine release, in both neuron-glial mixed cultures and primary microglia cultures. Finally, the conditioned medium of microglia pretreated with $\mathrm{OGD} / \mathrm{R}$ induced neuronal death, and the neuronal death was significantly inhibited by GPR 17 siRNA treatment. These findings suggest that GPR17 may mediate ischemia-like neuronal injury by regulating microglial activation in vitro.

\section{Acknowledgements}

Not applicable.

\section{Funding}

This study was supported by the National Natural Science Foundation of China (grant nos. 81401566 and 31301933) and the Zhejiang Provincial Natural Science Foundation (grant nos. LQ15H090005 and Y16H150006).

\section{Availability of data and materials}

The analyzed datasets generated during the study are available from the corresponding author on reasonable request.

\section{Authors' contributions}

BZ, EQW and QJS designed the research. BZ, QJS, HW, CXL, SWS, SHF performed experiments and data collection. BZ, HW and QJS analyzed the data and wrote the manuscript.

\section{Ethics approval and consent to participate}

Experimental protocols involving animals were approved by the Ethics Committee of Laboratory Animal Care and Welfare, School of Medicine, Zhejiang University (Hangzhou, China).

\section{Patient consent for publication}

Not applicable.

\section{Competing interests}

The authors declare that they have no competing interests.

\section{References}

1. Cekic C and Linden J: Purinergic regulation of the immune system. Nat Rev Immunol 16: 177-192, 2016.

2. Idzko M, Ferrari D and Eltzschig HK: Nucleotide signalling during inflammation. Nature 509: 310-317, 2014.

3. Ni NC, Yan D, Ballantyne LL, Barajas-Espinosa A, St Amand T, Pratt DA and Funk CD: A selective cysteinyl leukotriene receptor 2 antagonist blocks myocardial ischemia/reperfusion injury and vascular permeability in mice. J Pharmacol Exp Ther 339: 768-778, 2011.

4. Huang XQ, Zhang XY, Wang XR, Yu SY, Fang SH, Lu YB, Zhang WP and Wei EQ: Transforming growth factor $\beta 1$-induced astrocyte migration is mediated in part by activating 5-lipoxygenase and cysteinyl leukotriene receptor 1. J Neuroinflammation 9: 145, 2012.

5. Zhang XY, Wang XR, Xu DM, Yu SY, Shi QJ, Zhang LH, Chen L, Fang SH, Lu YB, Zhang WP and Wei EQ: HAMI 3379, a CysLT2 receptor antagonist, attenuates ischemia-like neuronal injury by inhibiting microglial activation. J Pharmacol Exp Ther 346: 328-341, 2013.

6. Shi QJ, Wang H, Liu ZX, Fang SH, Song XM, Lu YB, Zhang WP, Sa XY, Ying HZ and Wei EQ: HAMI 3379, a CysLT2R antagonist, dose- and time-dependently attenuates brain injury and inhibits microglial inflammation after focal cerebral ischemia in rats. Neuroscience 291: 53-69, 2015.

7. Ghosh A, Chen F, Thakur A and Hong H: Cysteinyl leukotrienes and their Receptors: Emerging therapeutic targets in central nervous system disorders. CNS Neurosci Ther 22: 943-951, 2016. 
8. Daniele S, Trincavelli ML, Gabelloni P, Lecca D, Rosa P, Abbracchio MP, and Martini C: Agonist-induced desensitization/resensitization of human $\mathrm{G}$ protein-coupled receptor 17: A functional cross-talk between purinergic and cysteinyl-leukotriene ligands. J Pharmacol Exp Ther 338: 559-567, 2011

9. Ciana P, Fumagalli M, Trincavelli ML, Verderio C, Rosa P, Lecca D, Ferrario S, Parravicini C, Capra V, Gelosa P, et al: The orphan receptor GPR 17 identified as a new dual uracil nucleotides/ cysteinyl-leukotrienes receptor. EMBO J 25: 4615-4627, 2006.

10. Temporini C, Ceruti S, Calleri E, Ferrario S, Moaddel R, Abbracchio MP and Massolini G: Development of an immobilized GPR17 receptor stationary phase for binding determination using frontal affinity chromatography coupled to mass spectrometry. Anal Biochem 384: 123-129, 2009.

11. Marucci G, Dal Ben D, Lambertucci C, Santinelli C, Spinaci A, Thomas A, Volpini R and Buccioni M: The G protein-coupled receptor GPR17: Overview and update. ChemMedChem 11: 2567-2574, 2016

12. Cosentino S, Castiglioni L, Colazzo F, Nobili E, Tremoli E, Rosa P, Abbracchio MP, Sironi L and Pesce M: Expression of dual nucleotides/cysteinyl-leukotrienes receptor GPR17 in early trafficking of cardiac stromal cells after myocardial infarction. J Cell Mol Med 18: 1785-1796, 2014.

13. Franke H, Parravicini C, Lecca D, Zanier ER, Heine C, Bremicker K, Fumagalli M, Rosa P, Longhi L, Stocchetti N, et al: Changes of the GPR 17 receptor, a new target for neurorepair, in neurons and glial cells in patients with traumatic brain injury. Purinergic Signal 9: 451-462, 2013.

14. Lecca D, Trincavelli ML, Gelosa P, Sironi L, Ciana P, Fumagalli M, Villa G, Verderio C, Grumelli C, Guerrini U, et al: The recently identified P2Y-like receptor GPR17 is a sensor of brain damage and a new target for brain repair. PLoS One 3 : e3579, 2008

15. Boda E, Viganò F, Rosa P, Fumagalli M, Labat-Gest V, Tempia F, Abbracchio MP, Dimou L and Buffo A: The GPR17 receptor in NG2 expressing cells: Focus on in vivo cell maturation and participation in acute trauma and chronic damage. Glia 59: 1958-1973, 2011

16. Ren H, Cook JR, Kon N and Accili D: Gpr17 in AgRP neurons regulates feeding and sensitivity to insulin and leptin. Diabetes 64: 3670-3679, 2015.

17. Zhao B, Zhao CZ, Zhang XY, Huang XQ, Shi WZ, Fang SH, Lu YB, Zhang WP, and Wei EQ: The new P2Y-like receptor $\mathrm{G}$ protein-coupled receptor 17 mediates acute neuronal injury and late microgliosis after focal cerebral ischemia in rats. Neuroscience 202: 42-57, 2012

18. Ceruti S, Villa G, Genovese T, Mazzon E, Longhi R, Rosa P, Bramanti P, Cuzzocrea S and Abbracchio MP: The P2Y-like receptor GPR17 as a sensor of damage and a new potential target in spinal cord injury. Brain 132: 2206-2218, 2009.

19. Fumagalli M, Lecca D and Abbracchio MP: CNS remyelination as a novel reparative approach to neurodegenerative diseases: The roles of purinergic signaling and the P2Y-like receptor GPR17. Neuropharmacology 104: 82-93, 2016.

20. Chen Y, Wu H, Wang S, Koito H, Li J, Ye F, Hoang J, Escobar SS Gow A, Arnett HA, et al: The oligodendrocyte-specific G protein-coupled receptor GPR17 is a cell-intrinsic timer of myelination. Nat Neurosci 12: 1398-1406, 2009.

21. Ou Z, Sun Y, Lin L, You N, Liu X, Li H, Ma Y, Cao L, Han Y Liu M, et al: Olig2-targeted G-protein-coupled receptor Gpr17 regulates oligodendrocyte survival in response to lysolecithin-induced demyelination. J Neurosci 36: 10560-10573, 2016.

22. Simon K, Hennen S, Merten N, Blättermann S, Gillard M, Kostenis E and Gomeza J: The orphan G protein-coupled receptor GPR17 negatively regulates oligodendrocyte differentiation via Gai/o and its downstream effector molecules. J Biol Chem 291: 705-718, 2016

23. Coppi E, Maraula G, Fumagalli M, Failli P, Cellai L, Bonfanti E, Mazzoni L, Coppini R, Abbracchio MP, Pedata F and Pugliese AM: UDP-glucose enhances outward $\mathrm{K}(+)$ currents necessary for cell differentiation and stimulates cell migration by activating the GPR 17 receptor in oligodendrocyte precursors. Glia 61: 1155-1171, 2013.

24. Meloni BP, Majda BT and Knuckey NW: Establishment of neuronal in vitro models of ischemia in 96-well microtiter strip-plates that result in acute, progressive and delayed neuronal death. Neuroscience 108: 17-26, 2001.

25. Zhao B, Zhang M, Han X, Zhang XY, Xing Q, Dong X, Shi QJ, Huang P, Lu YB, Wei EQ, et al: Cerebral ischemia is exacerbated by extracellular nicotinamide phosphoribosyltransferase via a non-enzymatic mechanism. PLoS One 8: e85403, 2013.
26. Huang XJ, Zhang WP, Li CT, Shi WZ, Fang SH, Lu YB, Chen Z and Wei EQ: Activation of CysLT receptors induces astrocyte proliferation and death after oxygen-glucose deprivation. Glia 56: 27-37, 2008

27. Ni M, and Aschner M: Neonatal rat primary microglia: Isolation, culturing, and selected applications. Curr Protoc Toxicol Chapter 12: Unit 12. 17, 2010.

28. Goldberg MP and Choi DW: Combined oxygen and glucose deprivation in cortical cell culture: Calcium-dependent and calcium-independent mechanisms of neuronal injury. J Neurosci 13: 3510-3524, 1993.

29. Daniele S, Lecca D, Trincavelli ML, Ciampi O, Abbracchio MP and Martini C: Regulation of PC12 cell survival and differentiation by the new P2Y-like receptor GPR17. Cell Signal 22: 697-706, 2010.

30. Qi LL, Lu YB, Shi WZ, Zhao CZ, Zhang YM, Chen LP, Zhang LH, Fang SH, Bao JF, Shen JG and Wei EQ: Preparation and identification of a polyclonal antibody against novel cysteinyl leukotriene receptor GPR17. Zhejiang Da Xue Xue Bao Yi Xue Ban 38: 357-361, 2009 (In Chinese).

31. Zhang Z, Luo J, Huang J, Liu Z, Fang S, Zhang WP, Wei E and Lu Y: Leukotriene D4 activates BV2 microglia in vitro. Zhejiang Da Xue Xue Bao Yi Xue Ban 42: 253-260, 2013 (In Chinese).

32. Neher JJ, Neniskyte U, Hornik T and Brown GC: Inhibition of $\mathrm{UDP} / \mathrm{P} 2 \mathrm{Y} 6$ purinergic signaling prevents phagocytosis of viable neurons by activated microglia in vitro and in vivo. Glia 62: 1463-1475, 2014.

33. Qi AD, Harden TK and Nicholas RA: Is GPR17 a P2Y/leukotriene receptor? examination of uracil nucleotides, nucleotide sugars, and cysteinyl leukotrienes as agonists of GPR17. J Pharmacol Exp Ther 347: 38-46, 2013.

34. Norregaard K, Benned-Jensen T and Rosenkilde MM: EBI2, GPR18 and GPR17-three structurally related, but biologically distinct 7TM receptors. Curr Top Med Chem 11: 618-628, 2011.

35. Benned-Jensen T and Rosenkilde MM: Distinct expression and ligand-binding profiles of two constitutively active GPR17 splice variants. Br J Pharmacol 159: 1092-1105, 2010.

36. Hennen S, Wang H, Peters L, Merten N, Simon K, Spinrath A, Blättermann S, Akkari R, Schrage R, Schröder R, et al: Decoding signaling and function of the orphan $\mathrm{G}$ protein-coupled receptor GPR17 with a small-molecule agonist. Sci Signal 6: ra93, 2013.

37. Simon K, Merten N, Schröder R, Hennen S, Preis P, Schmitt NK, Peters L, Schrage R, Vermeiren C, Gillard M, et al: The orphan receptor Gpr17 Is unresponsive to uracil-nucleotides and cysteinyl-leukotrienes. Mol Pharmacol 91: 518-532, 2017.

38. Boccazzi M, Lecca D, Marangon D, Guagnini F, Abbracchio MP and Ceruti S: A new role for the P2Y-like GPR17 receptor in the modulation of multipotency of oligodendrocyte precursor cells in vitro. Purinergic Signal 12: 661-672, 2016.

39. Harden TK: Enigmatic GPCR finds a stimulating drug. Sci Signal 6: pe34, 2013

40. Fumagalli M, Bonfanti E, Daniele S, Zappelli E, Lecca D, Martini C, Trincavelli ML and Abbracchio MP: The ubiquitin ligase $\mathrm{Mdm} 2$ controls oligodendrocyte maturation by intertwining mTOR with $\mathrm{G}$ protein-coupled receptor kinase 2 in the regulation of GPR17 receptor desensitization. Glia 63: 2327-2339, 2015.

41. Kim JY, Kim N and Yenari MA: Mechanisms and potential therapeutic applications of microglial activation after brain injury. CNS Neurosci Ther 21: 309-319, 2015

42. Ma Y, Wang J, Wang YA and Yang GY: The biphasic function of microglia in ischemic stroke. Prog Neurobiol 157: 247-272, 2017

43. Yang X, Lou Y, Liu G, Wang X, Qian Y, Ding J, Chen S and Xiao Q: Microglia P2Y6 receptor is related to Parkinson's disease through neuroinflammatory process. J Neuroinflammation 14: $38,2017$.

44. Block ML, Zecca L and Hong JS: Microglia-mediated neurotoxicity: Uncovering the molecular mechanisms. Nat Rev Neurosci 8: 57-69, 2007.

45. Koizumi S, Shigemoto-Mogami Y, Nasu-Tada K, Shinozaki Y, Ohsawa K, Tsuda M, Joshi BV, Jacobson KA, Kohsaka S and Inoue K: UDP acting at P2Y6 receptors is a mediator of microglial phagocytosis. Nature 446: 1091-1095, 2007.

46. Günther A, Manaenko A, Franke H, Dickel T, Berrouschot J, Wagner A, Illes P and Reinhardt R: Early biochemical and histological changes during hyperbaric or normobaric reoxygenation after in vitro ischaemia in primary corticoencephalic cell cultures of rats. Brain Res 946: 130-138, 2002.

47. Lazarowski ER, Shea DA, Boucher RC and Harden TK: Release of cellular UDP-glucose as a potential extracellular signaling molecule. Mol Pharmacol 63: 1190-1197, 2003. 
48. Kreda SM, Seminario-Vidal L, Heusden C and Lazarowski ER Thrombin-promoted release of UDP-glucose from human astrocytoma cells. Br J Pharmacol 153: 1528-1537, 2008.

49. Zhou Y, Wei EQ, Fang SH, Chu LS, Wang ML, Zhang WP, Yu GL, Ye YL, Lin SC and Chen Z: Spatio-temporal properties of 5-lipoxygenase expression and activation in the brain after focal cerebral ischemia in rats. Life Sci 79: 1645-1656, 2006.

50. Ciceri P, Rabuffetti M, Monopoli A and Nicosia S: Production of leukotrienes in a model of focal cerebral ischaemia in the rat. $\mathrm{Br}$ J Pharmacol 133: 1323-1329, 2001.

51. Maekawa A, Balestrieri B, Austen KF and Kanaoka Y: GPR17 is a negative regulator of the cysteinyl leukotriene 1 receptor response to leukotriene D4. Proc Natl Acad Sci USA 106: 11685-11690, 2009.

52. Maekawa A, Xing W, Austen KF and Kanaoka Y: GPR17 regulates immune pulmonary inflammation induced by house dust mites. J Immunol 185: 1846-1854, 2010.
53. Fang SH, Wei EQ, Zhou Y, Wang ML, Zhang WP, Yu GL, Chu LS and Chen Z: Increased expression of cysteinyl leukotriene receptor-1 in the brain mediates neuronal damage and astrogliosis after focal cerebral ischemia in rats. Neuroscience 140: 969-979, 2006.

54. Zhao CZ, Zhao B, Zhang XY, Huang XQ, Shi WZ, Liu HL, Fang SH, Lu YB, Zhang WP and Wei EQ: Cysteinyl leukotriene receptor 2 is spatiotemporally involved in neuron injury, astrocytosis and microgliosis after focal cerebral ischemia in rats. Neuroscience 189: 1-11, 2011

This work is licensed under a Creative Commons Attribution-NonCommercial-NoDerivatives 4.0 International (CC BY-NC-ND 4.0) License. 